\title{
Condutas em Atenção Primária: desenvolvendo um sumário online, em português, de prática clínica baseada em evidências
}

\author{
Condutas em Atenção Primária: developing an online evidence-based clinical practice summary \\ in Portuguese
}

\section{Condutas em Atenção Primária: desarrollo de un sumario en línea, en portugués, de la práctica clínica basada en evidencias}

Donavan de Souza Lúcioํㅜㄹ, Diogo Luis Scalco ${ }^{1}$

${ }^{1}$ Pesquisador independente.

\section{Resumo}

Introdução: Embora existam internacionalmente diversos sumários online baseados em evidências, a maioria é de língua inglesa, pago ou com restrições de acesso internacional. Desta forma, estamos desenvolvendo um sumário de prática clínica baseado em evidências, em português, gratuito, transparente e livre de insumos da indústria farmacêutica. Métodos: Selecionamos 28 tópicos para a primeira fase do projeto. Estes tópicos compõem problemas que abrangem $50 \%$ da demanda em atenção primária, são desenvolvidos sob a metodologia de revisões rápidas e submetidos à revisão por pares. A busca por evidência é realizada de forma discricionária, dando preferência às revisões sistemáticas. $O$ website é financiado pelos autores, os quais não possuem conflitos de interesse. O conteúdo do site está sob licença Creative Commons BY-NC-SA. Resultados: Criamos um manual para o desenvolvimento dos tópicos e o site www.condutas.com.br está disponível para acesso, com três tópicos desenvolvidos: resfriado comum, hipertensão arterial e pré-natal. Conclusão: O Condutas em Atenção Primária possui qualidade editorial e metodologia baseada em evidências adequada, se comparada a outros sumários online, o que é um avanço para a prática clínica nacional. Seu perfil inovador democratiza o acesso a informações atuais, consistentes e diversas, antes restritas ao leitor de língua inglesa, contudo, ao mesmo tempo enfrenta as limitações por seu pioneirismo e inerente amadorismo.

Palavras-chave: Medicina Baseada em Evidências; Tomada de Decisões; Guias de Prática Clínica como Assunto; Atenção Primária à Saúde

Como citar: Lúcio DS, Scalco DL. Condutas em Atenção Primária: desenvolvendo um sumário online, em português, de prática clínica baseada em evidências. Rev Bras Med Fam Comunidade. 2019;14(41):1707. https://doi.org/10.5712/rbmfc14(41)1707

\author{
Autor correspondente: \\ Donavan de Souza Lúcio. \\ E-mail: donavanlucio@gmail.com \\ Fonte de financiamento: \\ declaram não haver. \\ Parecer CEP: \\ não se aplica. \\ Procedência e revisão por pares: \\ revisado por pares. \\ Recebido em: 27/02/2018. \\ Aprovado em: 02/01/2019.
}




\begin{abstract}
Introduction: While there are, internationally, several evidence-based online summaries most are English-language, paid or internationally restricted. Thus, we are developing an online evidence-based point-of-care summary, in Portuguese, free of charge, transparent and free of pharmaceutical industry inputs. Methods: We selected 28 topics for the first phase of the project. These topics comprise problems that cover $50 \%$ of the demand in primary care, are developed under the methodology of rapid reviews, and are peer reviewed. We conduct the search for evidence in a discretionary manner, giving preference to systematic reviews. The authors, who have no conflicts of interests, fund the website. The content of the site is under Creative Commons BY-NC-SA license. Results: We created a manual for the development of the topics and the website www.condutas.com.br is available for access with three topics developed: resfriado comum (common cold), hipertensão arterial (hypertension) and pré-natal (antenatal care). Conclusion: The Condutas em Atenção Primária has editorial quality and adequate evidence-based methodology, compared to other online summaries, which is an advance for the Brazilian clinical practice. Its innovative profile democratizes access to current, consistent and diverse information previously restricted to the English-language reader, yet, at the same time, it faces the limitations of its pioneering and inherent amateurism.
\end{abstract}

Keywords: Evidence-Based Medicine; Decision Making; Practice Guidelines as Topic; Primary Health Care

\title{
Resumen
}

Introducción: Aunque existan internacionalmente diversos sumarios en línea basados en evidencias, la mayoría es de lengua inglesa, pago o con restricciones de acceso internacional. Así, estamos desarrollando un sumario de medicina basada en evidencias, accesible desde internet, en portugués, gratis, transparente y sin humus industriales. Métodos: Seleccionamos 28 temas para la primera fase del proyecto. Estos temas comprenden problemas que cubren el $50 \%$ de la demanda en atención primaria, se desarrollan bajo la metodología de revisiones rápidas y son sometidos a revisión por pares. Llevamos a cabo la búsqueda de pruebas de manera discrecional, dando preferencia a las revisiones sistemáticas. El sitio web es financiado por los autores, que no tienen conflictos de intereses. El contenido del sitio está bajo la licencia Creative Commons BY-NC-SA Resultados: Creamos un manual para el desarrollo de los temas y el sitio www.condutas.com.br está disponible para el acceso con tres temas desarrollados: resfriado comum (resfriado común), hipertensão arterial (hipertensión arterial) y pré-natal (prenatal). Conclusión: El Condutas em Atenção Primária tiene calidad editorial y metodología basada en evidencias adecuada si se compara a otros sumarios en línea, lo que es un avance a la práctica clínica brasileña. Su perfil innovador democratiza el acceso a informaciones actuales, coherentes y diversas, antes restringidas al lector en idioma inglés, sin embargo, al mismo tiempo enfrenta las limitaciones por su pionerismo e inherente inexperiencia.

Palabras clave: Medicina Basada en la Evidencia;Toma de Decisiones; Guías de Práctica Clínica como Asunto; Atención Primaria de Salud

\section{Introdução}

Durante um dia de trabalho surgem muitas questões clínicas e a maior parte delas permanece não resolvida ou esquecida ao final do dia. ${ }^{1}$ Embora atualmente existam diversos sumários online baseados em evidências, a maioria é de língua inglesa, pago ou com restrições de acesso internacional. ${ }^{2,3}$

No Brasil, o Ministério da Saúde, na tentativa de suprir esta carência, disponibiliza pelo Portal Saúde Baseado em Evidências desde 2012 acesso gratuito ao Dynamed ${ }^{\circledR}$. Dentre as ferramentas off-line baseadas em evidências com foco em atenção primária, destacam-se o livro "Medicina ambulatorial: condutas clínicas em atenção primária", de Bruce Duncan e colaboradores, editado desde 1996 e atualmente na quarta edição, ${ }^{4}$ e o Pratical Approach to Care Kit Brasil (PACK Brasil), realizado por meio de uma parceria entre o BMJ e o município de Florianópolis. ${ }^{5,6}$

Atentos à carência de um suporte de tomadas de decisões, adaptado à atenção primária brasileira e baseado em evidências, iniciamos o desenvolvimento de um sumário online, com tópicos prevalentes, estruturado por revisões rápidas, editados e revisados por médicos de família. ${ }^{7-9}$ 


\section{Métodos}

\section{Desenho do estudo}

Relato de experiência do desenvolvimento de um sumário online, em português, de prática clínica baseada em evidências.

\section{Seleção dos tópicos}

Nos últimos dez anos, apenas dois estudos brasileiros avaliaram os motivos das consultas na atenção primária utilizando mais de uma unidade de saúde de atenção primária: um realizado em Betim, ${ }^{10}$ com três equipes de saúde da família, e outro realizado em Florianópolis, ${ }^{11} \mathrm{com} 29$ equipes. Baseamos os tópicos prioritariamente no diagnóstico de demanda em Florianópolis devido à maior consistência do estudo (Tabela 1). ${ }^{11}$

Tabela 1. Seleção dos tópicos e seções para a primeira fase do projeto.

\begin{tabular}{|c|c|}
\hline Seções & Tópicos \\
\hline \multirow{3}{*}{ Cuidado preventivo } & Pré-natal \\
\hline & Contracepção \\
\hline & Rastreamento \\
\hline \multirow{2}{*}{ Saúde mental } & Transtornos de humor \\
\hline & Tabagismo \\
\hline \multirow{3}{*}{ Metabolismo } & Diabetes Mellitus \\
\hline & Hipotireoidismo \\
\hline & Obesidade \\
\hline \multirow{2}{*}{ Cardiovascular } & Hipertensão arterial \\
\hline & Risco cardiovascular e dislipidemia \\
\hline Dermatológico & Infecções fúngicas da pele \\
\hline \multirow{3}{*}{ Musculoesquelético } & Dor lombar \\
\hline & Dor articular \\
\hline & Dor muscular \\
\hline \multirow{4}{*}{ Olhos, ouvidos, boca e nariz } & Resfriado comum \\
\hline & Amigdalite aguda \\
\hline & Otite \\
\hline & Rinite alérgica \\
\hline \multirow{2}{*}{ Gastrointestinal } & Gastroenterite aguda \\
\hline & Dispepsia \\
\hline Respiratório & Asma \\
\hline \multirow{4}{*}{ Sintomas gerais } & Dor abdominal \\
\hline & Dor de cabeça \\
\hline & Prurido \\
\hline & Febre \\
\hline \multirow{2}{*}{ Geniturinário } & Infecção urinária \\
\hline & Infecções vaginais \\
\hline
\end{tabular}




\section{Desenvolvimento dos tópicos}

Cada tópico é desenvolvido por um ou dois editores, médicos de família, sob a metodologia de revisões rápidas. O desenvolvimento do tópico é baseado na resposta a perguntas clínicas que se encaixam nos domínios: prevenção primária, rastreamento, prognóstico, diagnóstico, terapêutica, danos e efeitos adversos, seguimento, encaminhamento e discussão. A formulação das perguntas é preferencialmente realizada antes da busca por evidências, e as perguntas são geralmente estruturadas com base no acrônimo PICO (Population/população, Intervention/intervenção, Comparison/comparação e Outcome/desfecho).

Ainda não há definição nem metodologias bem estabelecidas para revisões rápidas. ${ }^{9,12,13} \mathrm{Na}$ literatura disponível, o consenso é que a metodologia dessas revisões é mais sistemática e estruturada que as narrativas, e que o tempo de realização das revisões rápidas, quando comparadas às sistemáticas, é menor, muito embora as conclusões de ambas revisões sejam semelhantes. ${ }^{12,13}$ Entretanto, vale ressaltar que essa modalidade de revisão, quando comparada às revisões sistemáticas, tende a ser menos transparente, apresentando maior risco de viés e com conclusões menos sólidas. ${ }^{9,12}$

Os tópicos serão periodicamente atualizados em até cinco anos, ou antes caso surja uma nova evidência com potencial de mudar a prática clínica. Para a organização da construção e atualização dos tópicos, utilizamos o gerenciador de projetos Trello ${ }^{\circledR}$.

\section{Busca por evidência}

A busca por evidência é realizada de forma estruturada e hierárquica como segue:

\section{Primeiro passo}

Nesta etapa pesquisamos por revisões sistemáticas na Cochrane Library e recomendações sobre intervenções preventivas do US Preventive Services Task Force.

\section{Segundo passo}

Busca por recomendações em protocolos desenvolvidos sob avaliação crítica e sumários de evidência. Neste passo são utilizados protocolos do NICE Guidance e do National Guideline Clearinghouse (NGC) da Agency for Healthcare Research and Quality, e o sumário Dynamed ${ }^{\circledR}$, acessível pelo Portal Saúde Baseado em Evidências.

Neste passo é recomendado coletar apenas informações baseadas em revisões sistemáticas e fazer avaliação crítica das revisões. Frequentemente, apenas estudos primários estão disponíveis, o que torna inevitável utilizá-los.

\section{Terceiro passo}

Caso a busca pelas fontes acima tenham trazido resultados conflitantes, insatisfatórios ou se por outro motivo não possibilitaram responder à questão (eg. quais as indicações de encaminhamento para a atenção secundária ou terciária?), prosseguimos para as seguintes etapas: 
1. Busca por fontes primárias: TRIP Database ${ }^{\circledR}$ com filtro para evidência secundária, e PubMed (MEDLINE) com filtro para revisões sistemáticas, ou eventualmente ensaios clínicos randomizados e estudos observacionais.

2. Avaliação de consensos e prática usual: American Academy of Family Physicians; Canadian Medical Association; Royal College of General Practitioners; Royal Australian College of General Practitioners; Protocolos Clínicos e Diretrizes Terapêuticas; Protocolos de Encaminhamento da Atenção Básica para a Atenção Especializada; e Cadernos de Atenção Básica.

\section{Construção da recomendação}

A recomendação é composta pela própria recomendação e por sua sustentação. A recomendação é escrita em voz ativa e preferencialmente sem jargões. Para fins de transparência, indicamos entre colchetes, no início da sustentação, a fonte utilizada. A complexidade da construção da sustentação da recomendação varia conforme a necessidade do aprofundamento. No caso da utilização de fontes secundárias, como Dynamed $^{\circledR}$, a fonte primária citada é referenciada.

Exemplo:

\section{Questões:}

Qual o impacto do rastreamento de hipertrofia do ventrículo esquerdo em hipertensos? Quais os métodos de rastreamento de hipertrofia do ventrículo esquerdo em hipertensos? Qual o impacto do rastreamento de cardiopatias com eletrocardiograma em hipertensos?

\section{Sustentação:}

[Cochrane] Revisão "Pharmacotherapy for hypertension-induced left ventricular hypertrophy" em fase de protocolo (Finizola et al. 2016).

[USPSTF] Revisão de 2011 não recomenda o rastreamento de doença cardiovascular com eletrocardiograma de repouso ou esforço (a atualização está em andamento) (Chou et al. 2011). [NICE] NICE Guidance recomenda eletrocardiograma de repouso (NICE Guidance 2011).

[Dynamed: Hypertension] Revisão sistemática concluiu que a adoção de critérios para sobrecarga ventricular possui valor preditivo positivo suficiente para diagnosticar hipertrofia ventricular esquerda (HVE), mas valor preditivo negativo insuficiente (Pewsner et al. 2007). Em coorte, a redução da HVE associou-se a menor taxa de eventos cardiovasculares (Okin et al. 2004).

\section{Recomendação:}

Avalie solicitar eletrocardiograma de repouso.

\section{Graduação da recomendação}

Utilizamos a escala Strength of Recommendation Taxonomy (SORT), da American Academy of Family Physicians. ${ }^{14}$ foi desenvolvida para graduar estudos individuais ou no conjunto de evidência com ênfase em desfechos orientados ao paciente. 
A graduação das recomendações pela SORT possui três níveis:

A. Recomendação com evidência orientada ao paciente, consistente e de boa qualidade.

B. Recomendação com evidência orientada ao paciente, limitada ou inconsistente.

C. Recomendação baseada em consensos, prática usual, opinião, evidência orientada à doença, ou série de casos.

A graduação é colocada entre colchetes ao final da recomendação.

Exemplo:

Avalie solicitar eletrocardiograma de repouso [B]

A recomendação acima recebeu grau $B$, pois embora não tenha o suporte de ensaios clínicos randomizados, é baseada em estudo de coorte e orientada ao paciente.

\section{Sistema de revisão}

A revisão dos tópicos é realizada em duas fases: revisão interna e revisão por pares. A revisão interna é realizada pelos editores (quando houver mais de um). A revisão por pares é realizada por outros médicos de família. Em caso de reprovação na segunda fase, o tópico é reenviado à revisão interna. Após aprovação na revisão por pares o tópico é publicado.

\section{Declaração de conflitos de interesses}

É exigido dos editores e revisores dos tópicos declarar quaisquer conflitos de interesses existentes por meio do formulário desenvolvido pelo International Committee of Medical Journal Editors. ${ }^{15}$

\section{O website}

O website foi desenvolvido por Rafael Kupper de Oliveira Barros (programador e desenvolvedor web), construído em Bootstrap e PHP, e financiado pelos médicos de família Donavan de Souza Lúcio e Fernanda Karoline Melchior Silva Pinto, que não possuem conflitos de interesses. O domínio www.condutas.com.br foi registrado em 26 de novembro de 2016. O conteúdo do site está sob licença Creative Commons BY-NC-SA.

\section{Cronograma}

O desenvolvimento e testagem do site foram realizados de dezembro de 2016 a agosto de 2017. A finalização da primeira fase do projeto é prevista para o ano de 2019.

\section{Resultados}

Atualmente, o site www.condutas.com.br está disponível para acesso com três tópicos desenvolvidos: resfriado comum, hipertensão arterial e pré-natal. Sete tópicos estão em desenvolvimento e um aguarda revisão interna. Produzimos um manual para a construção dos tópicos que consta publicado como documento suplementar a este artigo. 


\section{Discussão}

Um grupo de estudiosos italianos vem avaliando, desde 2008, sumários online de prática clínica baseados em evidências. A metodologia utilizada envolve três dimensões de avaliação: a qualidade do processo editorial, a qualidade da metodologia baseada em evidências no desenvolvimento do conteúdo e o volume ou a amplitude das condições médicas abrangidas. ${ }^{3}$

A qualidade editorial é avaliada por cinco itens: autoria (reportar os autores de cada sumário), revisão (implementação de um processo de revisão por pares formal e estruturado), atualização (se os sumários foram atualizados ou revisados nos últimos dois anos), conflitos de interesse (reportar os conflitos de interesse de cada autor) e a existência ou não de suporte comercial. ${ }^{3}$

Aqualidade da metodologia baseada em evidências também foi avaliada por cinco itens: implementação de estratégia de busca de literatura ou vigilância para identificar informação atualizada, priorização de revisões sistemáticas sobre outras fontes, avaliação crítica, graduação formal da evidência e citação de opiniões de especialistas (separação de opiniões de especialista de outras fontes de evidência). ${ }^{3}$ Cada um dos itens dos dois domínios foi pontuado com três pontos se julgado "adequado", um se considerado que a informação "não estava clara" e zero pontos se "não adequado" ou "não reportado" ${ }^{3}$

De acordo com esta avaliação, ${ }^{3}$ o Condutas em Atenção Primária poderia ser avaliado como moderado, pontuando 12 de 15 pontos em qualidade editorial, pois não pontuaria em atualização (a mediana no estudo foi 12 pontos, variando de seis a 13 pontos), e pontuando 13 de 15 em qualidade da metodologia baseada em evidências, neste caso perderia pontos na descrição da avaliação crítica (a mediana do estudo foi 11 pontos, variando de quatro a 15 pontos). Na avaliação do volume provavelmente não pontuaria.

\section{Desafios}

O principal desafio está na sustentabilidade. O processo de desenvolvimento e publicação de cada tópico dura de um a três meses e, no momento, somos apenas dois editores. Após o termino da primeira fase, avaliaremos a criação de um aplicativo (pelos mesmos autores e desenvolvedores do website), provavelmente pago, que permita o acesso off-line ao conteúdo do site.

\section{Fortalezas}

A principal fortaleza, que também é o que nos motivou a iniciar e manter o projeto, é a gratuidade e o fato de ser um sumário em português. Atualmente, não há outro sumário online baseado em evidências no Brasil. Por ser virtual é facilmente atualizável e amplamente disponível. Está sob licença Creative Commons, o que permite ser replicado e ter suas informações utilizadas também para fins educacionais, diferentemente da maioria dos sumários existentes. Possui aplicabilidade na atenção primária e tem potencial de ser uma das fontes de referência em segunda opinião no Brasil. 


\section{Limitações}

A principal limitação está na própria metodologia. A pesquisa majoritariamente por fontes secundárias é potencialmente enviesada e o escasso número de revisores limita o refinamento do produto. Outra barreira são os artigos protegidos por paywall, obrigando o deslocamento para uma universidade pública para o acesso.

\section{Conclusão}

O Condutas em Atenção Primária possui qualidade editorial e metodologia baseada em evidências adequada, se comparada a outros sumários online, o que é um avanço para a prática clínica nacional. Seu perfil inovador democratiza o acesso a informações atuais, consistentes e diversas, antes restritas ao leitor de língua inglesa, contudo, ao mesmo tempo ainda enfrenta as limitações por seu pioneirismo e inerente amadorismo.

Concluímos, assim, que o uso, o investimento e manutenção de plataformas online permitem uma atualização clínica constante. Também cabe ressaltar a importância do uso de tecnologias leves como a decisão compartilhada que, além de colaborar para o enriquecimento da prática, provoca no usuário uma demanda por busca de fontes diversificadas, acendendo um olhar mais questionador, o que, consequentemente, qualifica a própria fonte.

\section{Conflito de interesses}

Declaram não haver.

\section{Referências}

1. Ebell MH. How to find answers to clinical questions. Am Fam Physician. 2009;79(4):293-6.

2. Banzi R, Liberati A, Moschetti I, Tagliabue L, Moja L. A Review of Online Evidence-based Practice Point-of-Care Information Summary Providers. J Med Internet Res [Internet]. 2010; [acesso 2016 Dez 8];12(3):e26. Disponível em: http://www.ncbi.nlm.nih.gov/pmc/articles/ PMC2956323/

3. Kwag KH, González-Lorenzo M, Banzi R, Bonovas S, Moja L. Providing Doctors With High-Quality Information: An Updated Evaluation of Web-Based Point-of-Care Information Summaries. J Med Internet Res [Internet]. 2016; [acesso 2016 Dez 8];18(1):e15. Disponível em: https://www.jmir.org/2016/1/e15/

4. Duncan BB, Schmidt MI, Giugliani ERJ, Duncan MS, Giugliani C. Medicina Ambulatorial: Condutas de Atenção Primária Baseadas em Evidências. $4^{\text {a }}$ ed. Porto Alegre: Artmed; 2014. 8178 p.

5. Fairall L, Bateman E, Cornick R, Faris G, Timmerman V, Folb N, et al. Innovating to improve primary care in less developed countries: towards a global model. BMJ Innov [Internet]. 2015; [acesso 2017 Abr 2];1(4):196-203. Disponível em: https://www.ncbi.nlm.nih.gov/pu bmed/?term=Fairall+L\%2C+Bateman+E\%2C+Cornick+R\%2C+Faris+G\%2C+Timmerman+V\%2C+Folb+N\%2C+et+al.+Innovating+to +improve+primary+care+in+less+developed+countries $\% 3 \mathrm{~A}+$ towards+a+global+model

6. Smith R. Being creative in developing primary care. The BMJ [Internet]. 2016; [acesso 2017 Abr 2]. Disponível em: http://blogs.bmj.com/ bmj/2016/09/26/richard-smith-being-creative-in-developing-primary-care/

7. Abou-Setta AM, Jeyaraman M, Attia A, Al-Inany HG, Ferri M, Ansari MT, et al. Methods for Developing Evidence Reviews in Short Periods of Time: A Scoping Review. PLoS One [Internet]. 2016; [acesso 2017 Abr 1];11(12):e0165903. Disponível em: http://journals.plos.org/ plosone/article?id=10.1371/journal.pone.0165903 
8. Ganann R, Ciliska D, Thomas H. Expediting systematic reviews: methods and implications of rapid reviews. Implement Sci [Internet]. 2010; [acesso 2017 Abr 2];5:56. https://doi.org/10.1186/1748-5908-5-56

9. Khangura S, Konnyu K, Cushman R, Grimshaw J, Moher D. Evidence summaries: the evolution of a rapid review approach. Syst Rev [Internet]. 2012; [acesso 2017 Abr 2];1:10. Disponível em: https://www.ncbi.nlm.nih.gov/pubmed/22587960

10. Landsberg GAP, Savassi LCM, Sousa AB, Freitas JMR, Nascimento JLS, Azagra R. Analysis of demand for family medical care in Brazil using the International Classification of Primary Care. Ciênc Saúde Coletiva [Internet]. 2012; [acesso 2016 Dez 8];17(11):3025-36. Disponível em: http://www.scielo.br/scielo.php?script=sci_arttext\&pid=S1413-81232012001100019

11. Gusso GDF. Diagnóstico de demanda em Florianópolis utilizando a Classificação Internacional de Atenção Primária: $2^{\underline{a}}$ edição (CIAP-2) [Tese de doutorado]. São Paulo: Universidade de São Paulo; 2009 [Internet]. [acesso 2016 Dez 8]. Disponível em: http://www.teses.usp. br/teses/disponiveis/5/5159/tde-08032010-164025/

12. Garritty C, Stevens A, Gartlehner G, King V, Kamel C. Cochrane Rapid Reviews Methods Group to play a leading role in guiding the production of informed high-quality, timely research evidence syntheses. Syst Rev [Internet]. 2016; [acesso 2017 Jul 18];5:184. https:// doi.org/10.1186/s13643-016-0360-z

13. Haby MM, Chapman E, Clark R, Barreto J, Reveiz L, Lavis JN. What are the best methodologies for rapid reviews of the research evidence for evidence-informed decision making in health policy and practice: a rapid review. Health Res Policy Syst [Internet]. 2016; [acesso 2017 Jul 18];14:83. https://doi.org/10.1186/s12961-016-0155-7

14. Ebell MH, Siwek J, Weiss BD, Woolf SH, Susman J, Ewigman B, et al. Strength of recommendation taxonomy (SORT): a patient-centered approach to grading evidence in the medical literature. Am Fam Physician. 2004;69(3):548-56. https://doi.org/10.3122/jabfm.17.1.59

15. International Committee of Medical Journal Editors. Conflicts of Interest [Internet]. [acesso 2019 Jan 13]. Disponível em: http://www.icmje. org/conflicts-of-interest/ 